\title{
Enhanced computed tomography imaging features predict tumor grade in pseudomyxoma peritonei
}

\author{
Pengtao Sun ${ }^{1}$, Xinbao $\mathrm{Li}^{2}$, Lingling Wang ${ }^{3}$, Rengui Wang ${ }^{1}$, Xuechao $\mathrm{Du}^{4} \wedge$ \\ ${ }^{1}$ Department of Radiology, Beijing Shijitan Hospital, Capital Medical University, Beijing, China; ${ }^{2}$ Department of Peritoneal Cancer Surgery, Beijing \\ Shijitan Hospital, Capital Medical University, Beijing, China; ${ }^{3}$ Department of Pathology, Beijing Shijitan Hospital, Capital Medical University, \\ Beijing, China; ${ }^{4}$ Department of Radiology, Beijing Chaoyang Hospital, Capital Medical University, Beijing, China
}

Contributions: (I) Conception and design: P Sun, X Du, R Wang; (II) Administrative support: X Li, L Wang, X Du; (III) Provision of study materials or patients: P Sun; (IV) Collection and assembly of data: P Sun, X Li, L Wang; (V) Data analysis and interpretation: P Sun, X Du; (VI) Manuscript writing: All authors; (VII) Final approval of manuscript: All authors.

Correspondence to: Rengui Wang. Department of Radiology, Beijing Shijitan Hospital, Capital Medical University, Yangfangdian Tieyi Road No. 10, Haidian District, Beijing 100038, China. Email: wangrg@bjsjth.cn; Xuechao Du. Department of Radiology, Beijing Chaoyang Hospital, Capital Medical University, Jingyuan Road No. 5, Shijingshan District, Beijing 100043, China. Email: duxuechao@ccmu.edu.cn.

Background: Because few studies have focused on the correlation between computed tomography (CT) signs and tumor grade in pseudomyxoma peritonei (PMP), we evaluated predictive value of abdominal enhanced CT in identifying high- $v s$. low-grade cases.

Methods: In all, 75 patients diagnosed with PMP after surgery were consecutively recruited. The preoperative enhanced CT images were retrospectively analyzed for ascites, hepatic scalloping, omental and peritoneal lesion appearance, intralesional calcification and septa, and peripheral organ involvement. Logistic regression models were applied to analyze the relationship of CT signs with PMP grade. Receiver operating characteristic curves were generated to evaluate the potential utility of CT signs in detecting high-grade PMP.

Results: Massive ascites $(\mathrm{P}=0.017)$ and peritoneal solid nodules $(\mathrm{P}<0.001)$ were more common in highgrade cases. Multivariate logistic regression identified massive ascites [odds ratio (OR) $=4.389,95 \%$ confidence interval (CI): 1.210-15.921; P=0.025] and peritoneal solid nodules (OR =19.932, 95\% CI: 3.560111.596; $\mathrm{P}<0.001)$ as independent predictors of high-grade PMP. For the 55 patients with hepatic scalloping, the maximum thickness of mucin deposition at the hepatic scalloping wave in high-grade PMP was thinner than that in low-grade PMP ( $\mathrm{P}=0.021)$. Thickness of mucin deposition at the hepatic scalloping wave (OR $=0.346,95 \%$ CI: 0.148-0.809; $\mathrm{P}=0.014)$ was an independent predictor of high-grade PMP, with a cutoff value of $18.6 \mathrm{~mm}$. Cancer antigen 125 (CA125) combined with CT signs was significantly better at diagnosing high-grade PMP than was CA125 alone in both the overall patients [area under the ROC curve (AUC): 0.812 vs. 0.656; $\mathrm{P}=0.020$ ] and those with hepatic scalloping (AUC: 0.859 vs. $0.600 ; \mathrm{P}=0.007$ ).

Conclusions: The CT signs of high-grade PMP significantly differ from those of low-grade PMP, and thus combining CT signs with CA125 may be highly valuable for classifying PMP.

Keywords: Computed tomography (CT); neoplasm grading; pseudomyxoma peritonei (PMP); scalloping

Submitted Oct 05, 2021. Accepted for publication Dec 22, 2021; Published online: 14 Jan 2022.

doi: 10.21037/qims-21-976

View this article at: https://dx.doi.org/10.21037/qims-21-976

\footnotetext{
^ ORCID: 0000-0002-5491-0398.
} 


\section{Introduction}

Pseudomyxoma peritonei (PMP) is a lesion formed by tumor cells that implant into the peritoneum and secrete mucus. PMP characteristically contains abundant jelly-like mucus and is extremely rare clinically, with an incidence of only 3.2 people per million (1).

The World Health Organization (WHO) divides PMP into low-grade and high-grade based on clinical progression and histopathological manifestations (2). The two subtypes differ in their treatment and prognosis. In general, PMP requires open cytoreductive surgery followed by hyperthermic intraperitoneal chemotherapy (3). However, low-grade PMP can be treated with less invasive laparoscopic cytoreductive surgery, with a recurrence rate roughly similar to that of open surgery (4). Moreover, the 5 -year survival rate for low-grade PMP is $63 \%$, compared with 23\% for high-grade PMP (5). Therefore, preoperative grading of PMP is critical to selecting the operative technique and predicting prognosis, but currently there is no effective preoperative clinical classification.

Enhanced computed tomography (CT) is a very important noninvasive method for predicting the histological grades of abdominal tumor (6) and is widely used to assess the extent of PMP (7). Previous studies have summarized the CT characteristics of PMP, which may help distinguish PMP from other peritoneal tumors (8). However, the predictive value of CT in identifying tumor grade in PMP has not been extensively explored. Because preoperative grading of PMP may estimate the aggressiveness of the tumor and provide valuable information for surgery, our study aimed to explore using CT examination for the differential diagnosis of high- versus low-grade cases. We present the following article in accordance with the Standards for Reporting of Diagnostic Accuracy (STARD) reporting checklist (available at https://qims.amegroups.com/article/view/10.21037/ qims-21-976/rc).

\section{Methods}

The study was conducted in accordance with the Declaration of Helsinki (as revised in 2013) and was approved by the Ethics Committee of Beijing Shijitan Hospital, Capital Medical University. Informed consent was waived because the study was a retrospective review. Patients diagnosed with PMP from January 2012 to September 2020 in Beijing Shijitan Hospital, Capital Medical University were enrolled consecutively from the hospital's electronic medical records. The inclusion criteria were the following: (I) PMP confirmed clinically and histologically, with histological review blindly reassessed by 1 experienced histopathologist; (II) preoperative contrastenhanced CT examination of the abdomen; and (III) a time interval between CT and operation of 3 months or less. The exclusion criteria were the following: (I) incomplete preoperative clinical data; (II) tumor treatment before CT examination, including cytoreductive surgery and chemotherapy; (III) mixed histopathological diagnosis containing both low-grade and high-grade components in the same patient; and (IV) poor CT images due to obvious respiratory artifacts. Finally, 75 PMP patients were included in this study. According to the WHO classification, patients were divided into low-grade and high-grade groups (2) and their demographic and clinical information [i.e., age, sex, and tumor markers, including cancer antigen (CA)125, CA19-9, carcinoembryonic antigen (CEA), and alphafetoprotein (AFP)] was recorded.

\section{CT protocol}

Abdominal enhanced CT was performed with a multislice spiral CT scanner (Revolution, GE Healthcare, Chicago, IL, USA; or Brilliance iCT, Philips Medical Healthcare, Amsterdam, The Netherlands). Scan parameters were as follows: $120 \mathrm{kVp} ; 100-250 \mathrm{~mA}$; pitch 1 ; and slice thickness and slice spacing, 2.5-3.0 mm. After unenhanced scanning, $80-100 \mathrm{~mL}$ of nonionic contrast agent (Iiopamid, $300 \mathrm{mgI} / \mathrm{mL}$, Bracco, Shanghai, China) was injected intravenously through the antecubital vein. Subsequently, the arterial phase, portal venous phase, and delayed phase were scanned for 30-40 s, 70-90 s, and 3 min after injection.

\section{CT image analysis}

All images were blindly and independently reassessed by 2 experienced radiologists. Any difference in diagnosis was resolved via discussion until a unified decision was reached.

CT image signs that need to be evaluated are outlined below:

(I) Presence of hepatic scalloping, measured according to the maximum thickness of mucin deposition at the hepatic scalloping wave (9).

(II) Involvement of peritoneum, defined as peritoneal thickening (>2 mm) (10), solid nodules, and cystic nodules-solid nodules were defined as soft tissue attenuation nodules with enhancement [increase 


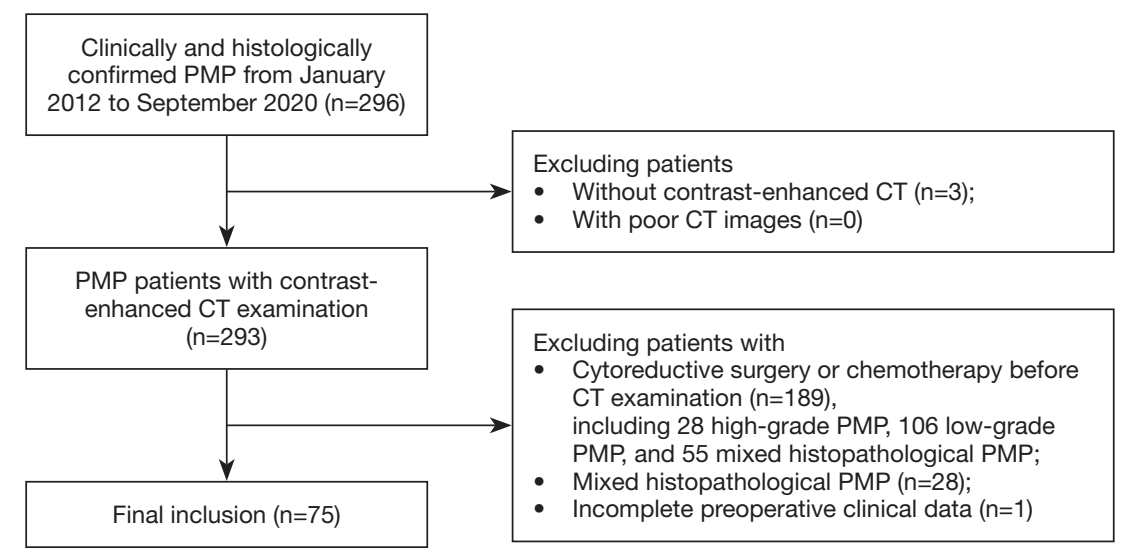

Figure 1 Flowchart of the study population. CT, computed tomography; PMP, pseudomyxoma peritonei.

in attenuation $\geq 20$ Hounsfield units (HU) after contrast] (11), and cystic nodules were defined as nodules without enhancement; patients with both solid and cystic nodules were classified as having solid nodules.

(III) Involvement of the omentum, including "smudged" appearance, cystic nodules, solid nodules, and omental cake.

(IV) Presence of calcification or septa.

(V) Presence of enlarged lymph nodes $(\geq 1 \mathrm{~cm})$ in the retroperitoneum or mesentery (12).

(VI) Presence of intestinal obstruction or ureteral involvement.

(VII) Abdominal ascites, defined as a small amount of ascites (effusion only in part of the viscera or peritoneal reflection) or massive ascites (effusion around all the viscera in the abdominal cavity, with organ displacement due to pressure).

\section{Statistical analysis}

Statistical analysis was performed using SAS version 9.1 (SAS, Cary, NC, USA) and SPSS version 26.0 (IBM Corp., Armonk, NY, USA). Quantitative data are represented as mean \pm standard deviation, or as median with interquartile range. Qualitative data are reported as counts and percentages. Intraclass correlation coefficient (ICC) was used to evaluate the repeatability of the maximum thickness of mucin deposition at the hepatic scalloping wave. Quantitative data were compared using independent samples $t$-test or Wilcoxon rank sum test, and qualitative indicators were compared using the chi-square test or Fisher's exact test. Univariate logistic regression was applied to analyze the relationship of various factors (including clinical data and CT signs) with tumor grade. Factors at a $\mathrm{P}$ value $<0.2$ in the univariate analysis were selected for the multivariate logistic regression model to screen for independent risk factors. The multivariate logistic regression model was constructed using the following formula: $\operatorname{logit}(\mathrm{P})=\ln$ [probability (event)/probability (no event)] $=\mathrm{a}+\mathrm{b}_{1}$ * variable $_{1}+\mathrm{b}_{2}{ }^{*}$ variable $_{2} \ldots$, a: constant, $\mathrm{b}_{1},{ }_{2}$ : coefficient. Probability was calculated as follows: probability $=1 /(1+$ $\mathrm{e}^{\text {-logit model }}$ ). Receiver operating characteristic (ROC) curves were used to find the optimal cutoff value of parameters in detecting high-grade PMP and to evaluate their predictive value. The ROC curves were based on logistic regression models incorporating significant clinical predictors with the CT signs. ROC was analyzed by MedCalc software (MedCalc Software Ltd., Flanders, Belgium). Statistical significance was defined as a two-sided $\mathrm{P}$ value $<0.05$.

\section{Results}

\section{Patients' clinical data}

Figure 1 displays the process of patient selection. We excluded those who did not undergo enhanced CT scanning, underwent cytoreductive surgery and chemotherapy before CT examination, were diagnosed as mixed histopathological PMP postoperation, or had incomplete clinical data. A total of 75 PMP patients were included, including 40 male and 35 female patients. Among the 75 cases, 57 were low-grade PMP and 18 were high-grade PMP (Table 1). CA19-9 and CA125 levels in high-grade PMP were significantly higher than those in low-grade PMP. No significant differences 
Table 1 Clinical and laboratory characteristics of the study population

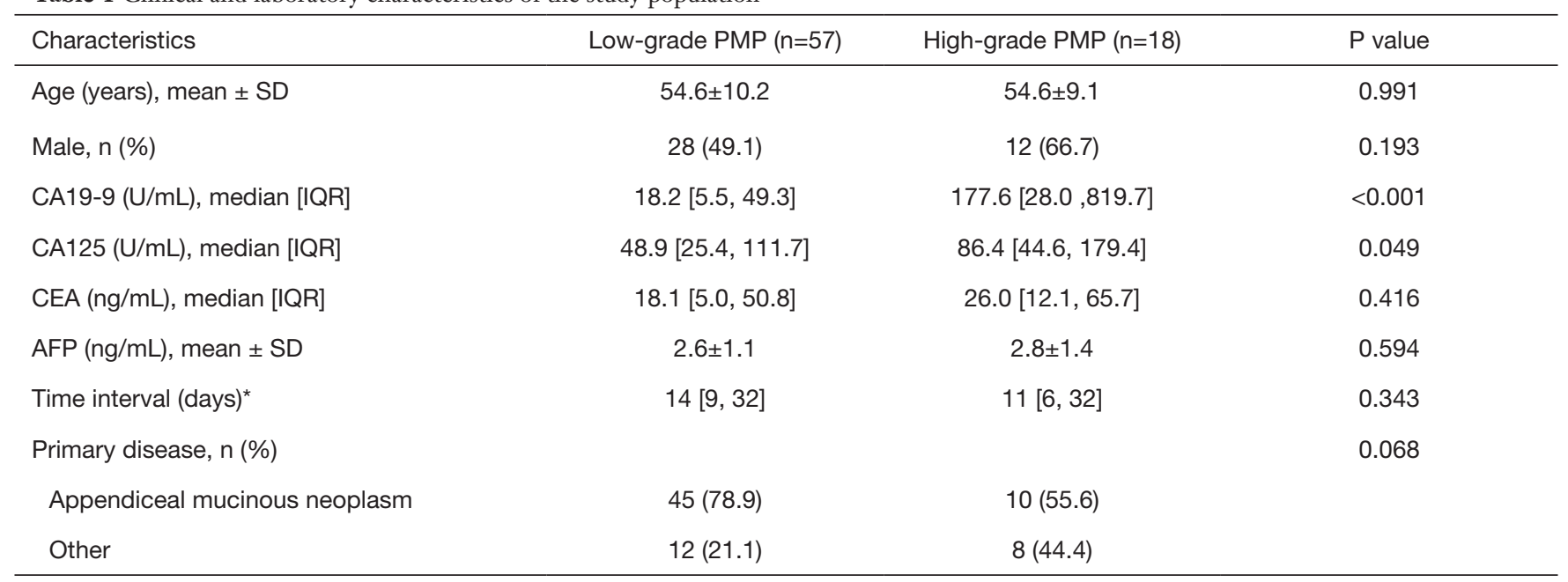

*, time interval between CT and operation. PMP, pseudomyxoma peritonei; SD, standard deviation; IQR, interquartile range; CA, cancer antigen; CEA, carcinoembryonic antigen; AFP, alpha-fetoprotein.

between the 2 groups were observed for age, sex, CEA, AFP, the time interval between CT and operation, or the primary disease of PMP.

\section{CT signs}

All 75 patients in this study tolerated enhanced CT without adverse events; a comparison of their CT signs is summarized in Table 2. Massive ascites was more common in high-grade PMP $(\mathrm{P}=0.017)$. Enlarged lymph nodes in the mesentery or retroperitoneum were detected in 3 high-grade patients, but not in any of the low-grade PMP cases $(\mathrm{P}=0.012)$. For the 55 patients with hepatic scalloping, the interrater ICC of the maximum thickness of mucin deposition at the hepatic scalloping wave was 0.969 $(\mathrm{P}<0.001)$. The maximum thickness of mucin deposition at the hepatic scalloping wave in high-grade PMP was thinner than that of low-grade $\mathrm{PMP}(\mathrm{P}=0.021$; Figure 2$)$.

Regarding the involvement of omentum, there was a difference in the involvement pattern of the omentum between the 2 groups $(\mathrm{P}=0.010)$. Omental solid nodules, which were detected only in the high-grade group, were significantly different between the 2 grades, while other omental involvement patterns showed no difference between the 2 grades.

For the involvement of the peritoneum, there was a difference in the manifestation of the peritoneum between the 2 grades $(\mathrm{P}<0.001)$. Patients with peritoneal solid nodules were more common in the high-grade PMP group, while patients with only peritoneal cystic nodules were more common in the low-grade PMP group (Figure 3). Other peritoneal involvement patterns showed no difference between the 2 grades.

No significant difference was found between the 2 grades in terms of the incidence of hepatic scalloping, septa or calcification, intestinal obstruction, or ureteral involvement.

\section{Risk factors for high-grade PMP}

CT imaging features associated with the grade of PMP are described in Table 3. Univariate logistic regression identified massive ascites [odds ratio $(\mathrm{OR})=3.750,95 \%$ confidence interval (CI): 1.221-11.518; $\mathrm{P}=0.021]$ and peritoneal solid nodules $(\mathrm{OR}=22.000,95 \% \mathrm{CI}: 4.061-119.186 ; \mathrm{P}<0.001)$ as predictors of high-grade PMP. However, the incidence of hepatic scalloping, omental cake, septa or calcification of the lesion, intestinal obstruction, and ureteral involvement were not risk factors. After adjusting for sex and CA125 (incorporated as a continuous variable), multivariate logistic regression highlighted massive ascites $(\mathrm{OR}=4.389,95 \% \mathrm{CI}$ : 1.210-15.921; $\mathrm{P}=0.025)$ and peritoneal solid nodules (OR $=19.932$, 95\% CI: $3.560-111.596, \mathrm{P}<0.001)$ as independent predictors of high-grade PMP (Table 4). A combined model for 75 patients was constructed based on CA125 and the CT signs according to the following formula: $\operatorname{logit}(\mathrm{P})=$ $-2.519+0.005$ * CA1 $25+2.776$ * peritoneal solid nodules + 1.145 * massive ascites, where $\mathrm{P}$ is the predicted probability of high-grade PMP. 
Table 2 Results of CT signs in different PMP grades

\begin{tabular}{|c|c|c|c|}
\hline Variables & Low-grade PMP $(n=57)$ & High-grade PMP $(n=18)$ & $P$ value \\
\hline Hepatic scalloping, n (\%) & $39(68.4)$ & $16(88.9)$ & 0.127 \\
\hline Thickness of mucin deposition (mm), median [IQR] & $20.2[10.6,25.1]$ & $11.1[5.5,17.7]$ & 0.021 \\
\hline Omental pattern, n (\%) & & & 0.010 \\
\hline Cystic nodules & $15(26.3)$ & $1(5.6)$ & \\
\hline Solid nodules & $0(0)$ & $2(11.1)$ & \\
\hline Cake & $23(40.4)$ & $12(66.7)$ & \\
\hline Peritoneal pattern, $\mathrm{n}(\%)$ & & & $<0.001$ \\
\hline Solid nodules & $2(3.5)$ & $8(44.4)$ & \\
\hline Septa, n (\%) & $9(15.8)$ & $1(5.6)$ & 0.435 \\
\hline Calcification, n (\%) & $12(21.1)$ & $8(44.4)$ & 0.068 \\
\hline Enlarged lymph nodes, $\mathrm{n}(\%)$ & $0(0)$ & $3(16.7)$ & 0.012 \\
\hline Bowel obstruction, n (\%) & $2(3.5)$ & $1(5.6)$ & 0.567 \\
\hline Ureteral involvement, $\mathrm{n}(\%)$ & $6(10.5)$ & $2(11.1)$ & 1.000 \\
\hline
\end{tabular}

CT, computed tomography; PMP, pseudomyxoma peritonei; IQR, interquartile range.
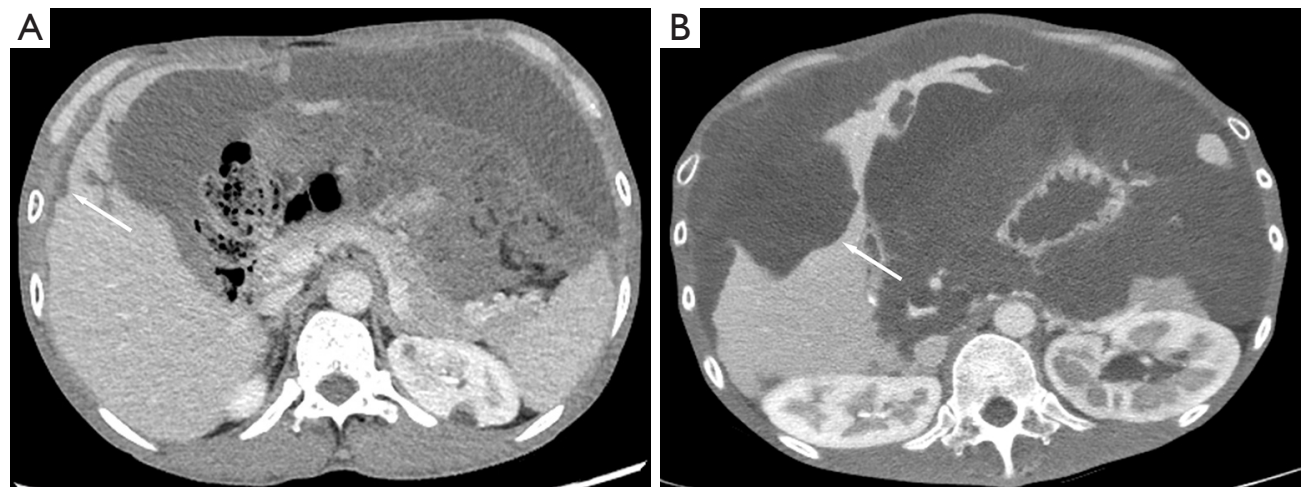

Figure 2 PMP with hepatic scalloping. (A) A 51-year-old male diagnosed with high-grade PMP. An axial image in the portal venous phase shows the maximum thickness of mucin deposition at the hepatic scalloping wave to be $5.4 \mathrm{~mm}$ (arrow). (B) A 44-year-old male diagnosed with low-grade PMP. Axial image in the portal venous phase shows the maximum thickness of mucin deposition at hepatic scalloping wave to be $78.7 \mathrm{~mm}$ (arrow). PMP, pseudomyxoma peritonei.

For the 55 patients with hepatic scalloping, univariate logistic regression identified the maximum thickness of mucin deposition at the hepatic scalloping wave as a predictor of high-grade $\mathrm{PMP}(\mathrm{OR}=0.427,95 \% \mathrm{CI}$ :
0.200-0.908; $\mathrm{P}=0.027)$. After adjusting for sex and CA125 (incorporated as a continuous variable), multivariate logistic regression underlined the maximum thickness of mucin deposition ( $\mathrm{OR}=0.346,95 \% \mathrm{CI}: 0.148-0.809 ; \mathrm{P}=0.014)$ as 

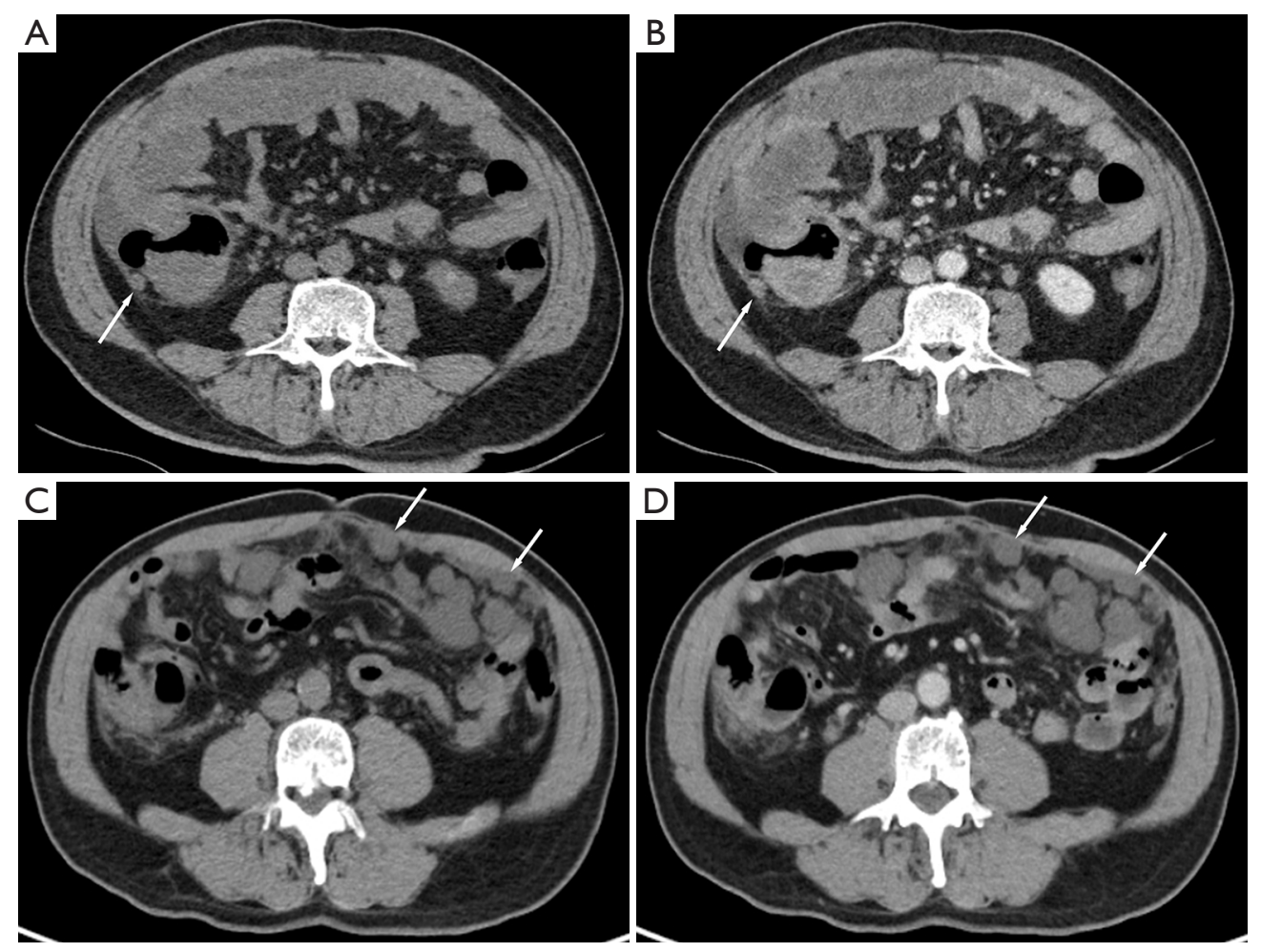

Figure 3 PMP with peritoneal nodules. (A,B) A 57-year-old male diagnosed with high-grade PMP. An enhanced solid nodule (arrows) can be seen on the peritoneum of the right para-colic gutter, with a CT attenuation of 29.5 HU on the unenhanced image (A) and 63.1 HU on the portal venous phase image (B). (C,D) A 64-year-old male diagnosed with low-grade PMP. Unenhanced cystic nodules of varying sizes (arrows) can be seen on the peritoneum, with a CT attenuation of $\approx 19.2 \mathrm{HU}$ on the unenhanced image $(\mathrm{C})$ and $\approx 23.4 \mathrm{HU}$ on the portal venous phase image (D). CT, computed tomography; HU, Hounsfield units; PMP, pseudomyxoma peritonei.

an independent predictor of high-grade PMP. A combined model for patients with hepatic scalloping was constructed based on CA125 and the CT according to the following formula: logit $(\mathrm{P})=-1.410+0.005 * \mathrm{CA} 125+2.763$ * peritoneal solid nodules +1.870 * massive ascites -0.801 * the maximum thickness of mucin deposition at hepatic scalloping wave, where $\mathrm{P}$ is the predicted probability of high-grade PMP.

\section{ROC curve analysis}

The area under the curve (AUC) of peritoneal solid nodules combined with massive ascites in diagnosing high-grade PMP was 0.759 (95\% CI: 0.647-0.851; $\mathrm{P}<0.001$ ), with sensitivity of $66.7 \%$ and specificity of $77.2 \%$. The AUC of CA125 in diagnosing high-grade PMP was 0.656 (95\% CI: $0.535-0.763 ; \mathrm{P}=0.024)$, with a cutoff value of $29.2 \mathrm{U} / \mathrm{mL}$, a sensitivity of $100.0 \%$, and a specificity of $30.9 \%$. The AUC of CA125 combined with CT signs (including peritoneal solid nodules and massive ascites) in diagnosing highgrade PMP was 0.812 (95\% CI: 0.704-0.894; $\mathrm{P}<0.001$ ), with a sensitivity of $77.8 \%$ and a specificity of $80.0 \%$. It was significantly better than that of CA125 alone $(\mathrm{P}=0.020$; Figure 4).

In the 55 patients with hepatic scalloping, the cutoff value of the thickness of mucin deposition at the hepatic scalloping wave in diagnosing high-grade PMP was 18.6 $\mathrm{mm}$, with an AUC of 0.700 , a sensitivity of $93.8 \%$, and a specificity of $51.3 \%$ (95\% CI: $0.562-0.816 ; \mathrm{P}=0.007$ ). CT signs (including thickness of mucin deposition, peritoneal solid nodules, and massive ascites) for diagnosing highgrade PMP had an AUC of 0.853 (95\% CI: 0.732-0.934; $\mathrm{P}<0.001$ ), a sensitivity of $87.5 \%$, and a specificity of $71.8 \%$. The AUC of CA125 for the diagnosis of high-grade PMP was 0.600 (95\% CI: 0.458-0.731; $\mathrm{P}=0.246$ ), with a sensitivity and specificity of $31.3 \%$ and $92.1 \%$, respectively. CA125 combined with CT signs (including thickness of mucin deposition, peritoneal solid nodules, and massive 
Table 3 Univariate logistic regression models for high-grade PMP

\begin{tabular}{|c|c|c|c|}
\hline Variables & OR & $95 \% \mathrm{Cl}$ & $P$ value \\
\hline Male & 2.071 & $0.683-6.280$ & 0.198 \\
\hline CA19-9 & 1.000 & $1.000-1.001$ & 0.240 \\
\hline CA125 & 1.004 & $0.999-1.008$ & 0.112 \\
\hline AFP & 1.137 & $0.715-1.806$ & 0.588 \\
\hline Massive ascites & 3.750 & $1.221-11.518$ & 0.021 \\
\hline Hepatic scalloping & 3.691 & $0.766-17.784$ & 0.104 \\
\hline Thickness of mucin deposition & 0.427 & $0.200-0.908$ & 0.027 \\
\hline Peritoneal pattern & 0.836 & $0.486-1.437$ & 0.517 \\
\hline Peritoneal solid nodules & 22.000 & $4.061-119.186$ & $<0.001$ \\
\hline Septa & 0.314 & $0.037-2.663$ & 0.288 \\
\hline Calcification & 3.000 & $0.972-9.258$ & 0.056 \\
\hline Bowel obstruction & 1.618 & $0.138-18.964$ & 0.701 \\
\hline Ureteral involvement & 1.063 & $0.195-5.793$ & 0.944 \\
\hline
\end{tabular}

$\mathrm{Cl}$, confidence interval; OR, odds ratio; PMP, pseudomyxoma peritonei; CA, cancer antigen; CEA, carcinoembryonic antigen; AFP, alphafetoprotein.

Table 4 Multivariate logistic regression models for high-grade PMP

\begin{tabular}{lccc}
\hline Variables & OR & $95 \% \mathrm{Cl}$ & P value \\
\hline Massive ascites & 4.389 & $1.210-15.921$ & 0.025 \\
Hepatic scalloping & 2.911 & $0.580-14.616$ & 0.194 \\
Thickness of mucin deposition & 0.346 & $0.148-0.809$ & 0.014 \\
Omental cake & 2.358 & $0.707-7.868$ & 0.163 \\
Peritoneal solid nodules & 19.932 & $3.560-111.596$ & $<0.001$ \\
Calcification & 2.482 & $0.773-7.972$ & 0.127 \\
\hline
\end{tabular}

$\mathrm{Cl}$, confidence interval; OR, odds ratio; PMP, pseudomyxoma peritonei.

ascites) for diagnosing high-grade PMP had an AUC of 0.859 (95\% CI: $0.737-0.938 ; \mathrm{P}<0.001$ ), a sensitivity of $87.5 \%$, and a specificity of $73.7 \%$, which was significantly better than that of CA125 alone ( $\mathrm{P}=0.007$; Figure 5).

\section{Discussion}

In this study, peritoneal solid nodules and massive ascites were found to be independent predictors of high-grade PMP. For patients with hepatic scalloping, the maximum thickness of mucin deposition at the hepatic scalloping wave was significantly thicker in low-grade PMP. The maximum thickness of mucin deposition at the hepatic scalloping wave was an independent predictor of high-grade PMP. Moreover, enlarged abdominal lymph nodes and solid nodules of the omentum could be detected only in high- 


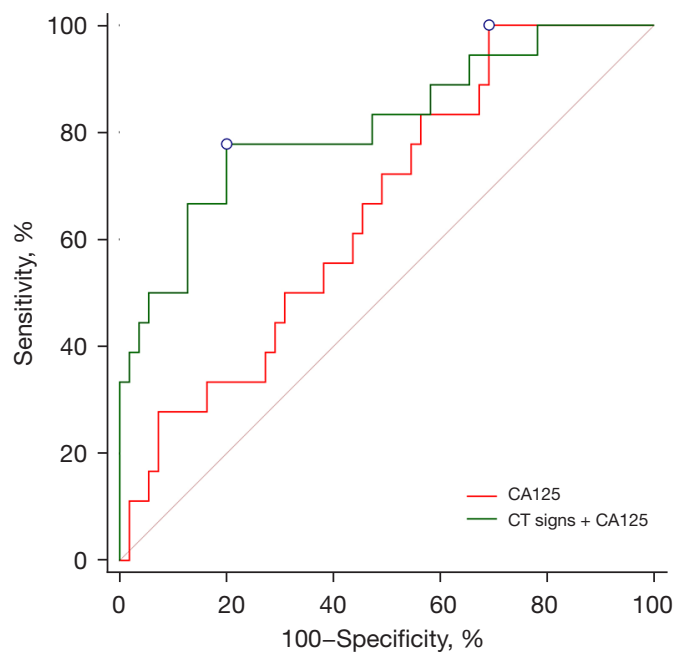

Figure 4 ROC in discriminating high- vs. low-grade PMP. The AUC increased from 0.656 to 0.812 when CA125 was combined with CT signs including peritoneal solid nodules and massive ascites $(\mathrm{P}=0.020)$. AUC, area under the curve; CT, computed tomography; PMP, pseudomyxoma peritonei; ROC, receiver operating characteristic; CA125, cancer antigen 125.

grade PMP. No significant difference was found between the 2 groups in terms of the incidence of hepatic scalloping and omental cake.

PMP has no specific clinical signs or symptoms. Nonspecific findings of abdominal distension and bowel obstruction can occur with disease progression (13). Preoperative grading is essential, but clinical indicators cannot effectively predict the grade of PMP. In this study, although CA125 was higher in high-grade PMP with an AUC of 0.656 , it had little value in distinguishing the different grades of PMP. However, the AUC of CT signs including peritoneal solid nodules and massive ascites combined with CA125 in diagnosing high-grade PMP was 0.812 , with high differential diagnostic value for high-grade PMP. This is important for guiding the choice of surgical approach.

Hepatic scalloping is a specific CT feature of PMP (14) and has great value in distinguishing PMP from other peritoneal diseases. Moreover, preoperative hepatic scalloping thickness can predict postoperative tumor recurrence in PMP patients (9). In our study, the incidence of hepatic scalloping in high- and low-grade PMP was similar, which is consistent with previous studies (9). However, compared with that of low-grade PMP, the maximum thickness of mucin deposition at the hepatic

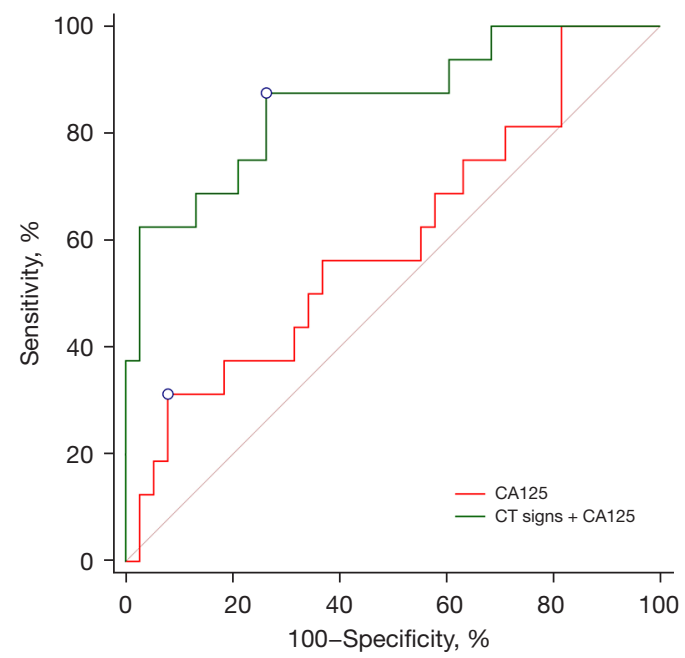

Figure 5 ROC for patients with hepatic scalloping in discriminating high- vs. low-grade PMP. The AUC increased from 0.600 to 0.859 when CA125 was combined with CT signs including the maximum thickness of mucin deposition at the hepatic scalloping wave, peritoneal solid nodules, and massive ascites $(\mathrm{P}=0.007)$. AUC, area under the curve; CT, computed tomography; PMP, pseudomyxoma peritonei; ROC, receiver operating characteristic; CA125, cancer antigen 125.

scalloping wave of high-grade PMP was thinner. The cutoff value of the maximum thickness of mucin deposition was $18.6 \mathrm{~mm}$, with an AUC of 0.700. It was previously reported that hepatic scalloping of high-grade PMP is mostly minimal while that of low-grade PMP is generally deeper (15), which also supports our conclusion. A potential explanation for the difference in thickness of mucin between the 2 groups might lie in differences in the composition of tumor cells. High-grade PMP tumor cells exhibit obvious atypia, whereas low-grade PMP tumor cells are mainly columnar epithelial cells, which may have vigorous mucus secretory function (16). This results in a more obvious thickness of the mucin deposition in low-grade PMP.

It is generally reported that PMP peritoneal implants are cystic lesions $(17,18)$. However, one case report showed that PMP could also manifest as a cystic-solid mass (19). Our study showed that PMP peritoneal lesions could be solid nodules. Moreover, patients with solid nodules were more likely to have high-grade PMP, whereas patients with only cystic nodules were more likely to have low-grade PMP. It is possible that high-grade PMP tumors have relatively more cellular components (20) due to their multilayered arrangement and active proliferation. In contrast, low-grade 
PMP tumor cells are generally arranged in a monolayer (20). Additionally, due to poor mucus secretion, high-grade PMP tumors may contain tiny cystic nodules that cannot be detected on CT images. These possibilities should be confirmed in further research.

Ronnet et al. divided PMP into disseminated peritoneal adenomucinosis (DPAM), peritoneal mucinous carcinomatosis (PMCA), and PMCA with intermediate (well-differentiated) features (PMCA-I) (21), but there was no significant difference between DPAM and PMCA-I in the 1-, 3-, and 5-year survival rates, respectively, with the prognosis of PMCA being significantly poor (22). Therefore, DPAM and PMCA-I were reclassified as low-grade PMP while PMCA was classified high-grade PMP (22); this system has since been adopted by the WHO (2). One study using the Ronnet classification reported no omental cake in low-grade PMP, but there were no PMCA-I cases in this study (15). Another study based on preoperative ultrasound found that both PMCA-I and PMCA had omental cake (23), indicating that it could form in low-grade PMP. In a study of 54 patients, 25 (46.3\%) and $29(53.7 \%)$ patients were diagnosed with high- and lowgrade PMP, respectively, and omental cake was detected in $41(85.0 \%)$ patients during surgery (24), which also suggests that omental cake can be present in low-grade PMP. In line with this, our study showed that both low-grade PMP and high-grade PMP could show omental cake. The incidence of omental cake in high-grade PMP (66.7\%) tended to be higher than in low-grade (40.4\%), but this difference was not statistically significant.

Massive ascites in our study was more common in highgrade PMP, which is inconsistent with previous studies (15). Accumulation of ascites may be attributed to increased vascular permeability induced by cytokines, or blockage of lymphatic vessels (25). High-grade PMP could produce more ascites because (I) compared with low-grade PMP, high-grade PMP tumor cells release more cytokines, such as tumor necrosis factor- $\alpha$ (26), causing increased vascular permeability (27); and (II) enlarged abdominal lymph nodes, a reliable sign of lymph node involvement, can be only detected in high-grade PMP (15), and lymph node involvement is highly associated with lymphatic tumor thrombus formation $(28,29)$, which results in the inability to remove excess fluid.

Our study has some limitations. First, the latest PMP pathological classification is based on the Peritoneal Surface Oncology Group International (PSOGI) classification, which divides PMP into 4 subtypes: acellular mucin, low- grade mucinous carcinoma peritonei or DPAM, highgrade mucinous carcinoma peritonei or PMCA, high-grade mucinous carcinoma peritonei with signet ring cells or peritoneal mucinous carcinomatosis with signet ring cells (PMCA-S) (30). Because only 5 cases of PMCA-S were included in our study and the PSOGI classification may not provide a better prognostic stratification than the WHO classification (31), we used the latter. Second, this was a retrospective study in a single institution. The potential value of abdominal enhanced CT in identifying high-grade PMP needs to be further confirmed by prospective studies with large sample sizes. Third, only the CT characteristics for pure high- or low-grade PMP were summarized; patients with mixed histological PMP were not included. Among the excluded mixed histopathological PMP patients, most had different pathological grades in different lesions. The CT signs of these patients have little value in distinguishing high- from low-grade because they may overlap with those of both high- and low-grade patients. Further research based on lesions rather than on patients is needed to confirm whether the conclusions of our study are applicable to mixed histopathological PMP patients.

In summary, peritoneal solid nodules and massive ascites are independent predictors of high-grade PMP. For patients with hepatic scalloping, those with a diagnosis of highgrade PMP have thinner mucin deposition. In contrast, the presence of hepatic scalloping, intralesional calcification, septal and peripheral organ involvement is of little diagnostic utility. Rather, CT signs combined with CA125 was found to be highly valuable for PMP classification.

\section{Acknowledgments}

Funding: None.

\section{Footnote}

Reporting Checklist: The authors have completed the STARD reporting checklist. Available at https://qims.amegroups. com/article/view/10.21037/qims-21-976/rc

Conflicts of Interest: All authors have completed the ICMJE uniform disclosure form (available at https://qims. amegroups.com/article/view/10.21037/qims-21-976/coif). The authors have no conflicts of interest to declare.

Ethical Statement: The authors are accountable for all aspects of the work in ensuring that questions related 
to the accuracy or integrity of any part of the work are appropriately investigated and resolved. The study was conducted in accordance with the Declaration of Helsinki (as revised in 2013). The study was approved by the Ethics Committee of Beijing Shijitan Hospital, Capital Medical University, and informed consent was waived because the study was retrospective review.

Open Access Statement: This is an Open Access article distributed in accordance with the Creative Commons Attribution-NonCommercial-NoDerivs 4.0 International License (CC BY-NC-ND 4.0), which permits the noncommercial replication and distribution of the article with the strict proviso that no changes or edits are made and the original work is properly cited (including links to both the formal publication through the relevant DOI and the license). See: https://creativecommons.org/licenses/by-nc-nd/4.0/.

\section{References}

1. Patrick-Brown TDJH, Carr NJ, Swanson DM, Larsen S, Mohamed F, Flatmark K. Estimating the Prevalence of Pseudomyxoma Peritonei in Europe Using a Novel Statistical Method. Ann Surg Oncol 2021;28:252-7.

2. Bosman FT, Carneiro F, Hruban RH, Theise ND. WHO classification of tumors of the digestive system. 4th edition. Lyon: International Agency for Research on Cancer Publisher, 2010.

3. Li Y, Yu Y, Liu Y. Report on the 9(th) International Congress on Peritoneal Surface Malignancies. Cancer Biol Med 2014;11:281-4.

4. Mercier F, Jeremie G, Alyami M, Delphine V, Vahan K, Pascal R, Sylvie I, Guillaume P, Olivier G. Long-term results of laparoscopic cytoreductive surgery and HIPEC for the curative treatment of low-grade pseudomyxoma peritonei and multicystic mesothelioma. Surg Endosc 2020;34:4916-23.

5. Carr NJ, Finch J, Ilesley IC, Chandrakumaran K, Mohamed F, Mirnezami A, Cecil T, Moran B. Pathology and prognosis in pseudomyxoma peritonei: a review of 274 cases. J Clin Pathol 2012;65:919-23.

6. Zhou Z, Lu J, Morelli JN, Hu D, Li Z, Xiao P, Hu X, Shen Y. Utility of noncontrast MRI in the detection and risk grading of gastrointestinal stromal tumor: a comparison with contrast-enhanced CT. Quant Imaging Med Surg 2021;11:2453-64.

7. Bouquot M, Dohan A, Gayat E, Barat M, Glehen O, Pocard M, Rousset P, Eveno C. Prediction of Resectability in Pseudomyxoma Peritonei with a New CT Score. Ann Surg Oncol 2018;25:694-701.

8. Diop AD, Fontarensky M, Montoriol PF, Da Ines D. CT imaging of peritoneal carcinomatosis and its mimics. Diagn Interv Imaging 2014;95:861-72.

9. Hotta M, Minamimoto R, Gohda Y, Tajima T, Kiyomatsu T, Yano H. Pseudomyxoma peritonei: visceral scalloping on CT is a predictor of recurrence after complete cytoreductive surgery. Eur Radiol 2020;30:4193-200.

10. Liang YF, Zheng GQ, Chen YF, Song H, Yin WJ, Zhang L. CT differentiation of diffuse malignant peritoneal mesothelioma and peritoneal carcinomatosis. J Gastroenterol Hepatol 2016;31:709-15.

11. Herts BR, Silverman SG, Hindman NM, Uzzo RG, Hartman RP, Israel GM, Baumgarten DA, Berland LL, Pandharipande PV. Management of the Incidental Renal Mass on CT: A White Paper of the ACR Incidental Findings Committee. J Am Coll Radiol 2018;15:264-73.

12. Hadley DA, Stephenson RA, Samlowski WE, Dechet CB. Patterns of enlarged lymph nodes in patients with metastatic renal cell carcinoma. Urol Oncol 2011;29:751-5.

13. Rizvi SA, Syed W, Shergill R. Approach to pseudomyxoma peritonei. World J Gastrointest Surg 2018;10:49-56.

14. Fonseca C, Carvalho S, Cunha TM, Gil RT, Abecasis N. The many faces of pseudomyxoma peritonei: a radiological review based on 30 cases. Radiol Bras 2019;52:372-7.

15. Bechtold RE, Chen MY, Loggie BW, Jackson SL, Geisinger K. CT appearance of disseminated peritoneal adenomucinosis. Abdom Imaging 2001;26:406-10.

16. Shetty S, Natarajan B, Thomas P, Govindarajan V, Sharma P, Loggie B. Proposed classification of pseudomyxoma peritonei: influence of signet ring cells on survival. Am Surg 2013;79:1171-6.

17. Pickhardt PJ, Perez AA, Elmohr MM, Elsayes KM. CT imaging review of uncommon peritoneal-based neoplasms: beyond carcinomatosis. Br J Radiol 2021;94:20201288.

18. Arraiza M, Metser U, Vajpeyi R, Khalili K, Hanbidge A, Kennedy E, Ghai S. Primary cystic peritoneal masses and mimickers: spectrum of diseases with pathologic correlation. Abdom Imaging 2015;40:875-906.

19. Liang L, Zhou N, Xu H, Liu D, Lu Y, Li F, Guo J. Urachal mucinous adenocarcinoma with pseudomyxoma peritonei: A case report. Medicine (Baltimore) 2017;96:e7548.

20. Carr NJ, Bibeau F, Bradley RF, Dartigues P, Feakins RM, Geisinger KR, Gui X, Isaac S, Milione M, Misdraji J, Pai RK, Rodriguez-Justo M, Sobin LH, van Velthuysen MF, Yantiss RK. The histopathological classification, 
diagnosis and differential diagnosis of mucinous appendiceal neoplasms, appendiceal adenocarcinomas and pseudomyxoma peritonei. Histopathology 2017;71:847-58.

21. Ronnett BM, Zahn CM, Kurman RJ, Kass ME, Sugarbaker PH, Shmookler BM. Disseminated peritoneal adenomucinosis and peritoneal mucinous carcinomatosis. A clinicopathologic analysis of 109 cases with emphasis on distinguishing pathologic features, site of origin, prognosis, and relationship to "pseudomyxoma peritonei". Am J Surg Pathol 1995;19:1390-408.

22. Bradley RF, Stewart JH 4th, Russell GB, Levine EA, Geisinger KR. Pseudomyxoma peritonei of appendiceal origin: a clinicopathologic analysis of 101 patients uniformly treated at a single institution, with literature review. Am J Surg Pathol 2006;30:551-9.

23. Li Y, Guo A, Tang J, Wang L, Wang J, Yu D. Role of preoperative sonography in the diagnosis and pathologic staging of pseudomyxoma peritonei. J Ultrasound Med 2013;32:1565-70.

24. Krause J, Bergman A, Graf W, Nilsson A, Mahteme H. Ultrasonography findings and tumour quantification in patients with pseudomyxoma peritonei. Eur J Radiol 2012;81:648-51.

25. Kuracha MR, Thomas P, Loggie BW, Govindarajan V. Patient-derived xenograft mouse models of pseudomyxoma peritonei recapitulate the human inflammatory tumor microenvironment. Cancer Med 2016;5:711-9.

26. Vlaeminck-Guillem V, Bienvenu J, Isaac S, Grangier B, Golfier F, Passot G, Bakrin N, Rodriguez-Lafrasse C, Gilly FN, Glehen O. Intraperitoneal cytokine level in

Cite this article as: Sun P, Li X, Wang L, Wang R, Du X. Enhanced computed tomography imaging features predict tumor grade in pseudomyxoma peritonei. Quant Imaging Med Surg 2022;12(4):2321-2331. doi: 10.21037/qims-21-976 patients with peritoneal surface malignancies. A study of the RENAPE (French Network for Rare Peritoneal Malignancies). Ann Surg Oncol 2013;20:2655-62.

27. Sethu S, Pushparaj PN, Melendez AJ. Phospholipase D1 mediates TNFalpha-induced inflammation in a murine model of TNFalpha-induced peritonitis. PLoS One 2010;5:e10506.

28. Moriya H, Ohbu M, Kobayashi N, Tanabe S, Katada N, Futawatari N, Sakuramoto S, Kikuchi S, Okayasu I, Watanabe M. Lymphatic tumor emboli detected by D2-40 immunostaining can more accurately predict lymph-node metastasis. World J Surg 2011;35:2031-7.

29. Liu X, Long Z, Cai H, Huang H, Shi Y, Wang Y. Analysis of lymph node metastasis correlation with prognosis in patients with T2 gastric cancer. PLoS One 2014;9:e105112.

30. Carr NJ, Cecil TD, Mohamed F, Sobin LH, Sugarbaker PH, González-Moreno S, Taflampas P, Chapman S, Moran BJ; Peritoneal Surface Oncology Group International. A Consensus for Classification and Pathologic Reporting of Pseudomyxoma Peritonei and Associated Appendiceal Neoplasia: The Results of the Peritoneal Surface Oncology Group International (PSOGI) Modified Delphi Process. Am J Surg Pathol 2016;40:14-26.

31. Baratti D, Kusamura S, Milione M, Bruno F, Guaglio M, Deraco M. Validation of the Recent PSOGI Pathological Classification of Pseudomyxoma Peritonei in a SingleCenter Series of 265 Patients Treated by Cytoreductive Surgery and Hyperthermic Intraperitoneal Chemotherapy. Ann Surg Oncol 2018;25:404-13. 